\title{
Right Ovarian Vein Thrombophlebitis Complicated by an Inferior Endocaval Floating Thrombus Revealed by Postpartum Pyrexia: A Case Report
}

\section{Faidherbe $\mathrm{C}^{\star}$}

Centre Hospitalier de Wallonie Picarde (CHwapi), Service des Urgences, Tournai, Belgium

${ }^{*}$ Corresponding author: Faidherbe C, Centre Hospitalier de Wallonie Picarde (CHwapi), site Union, Service des Urgences, Rue des Sports 51, 7500 Tournai, Belgium, Tel: 0032460949681, E-mail: cfaidherbe@yahoo.fr

Citation: Faidherbe C (2021) Right Ovarian Vein Thrombophlebitis Complicated by an Inferior Endocaval Floating Thrombus Revealed by Postpartum Pyrexia: A Case Report. J Case Rep Stud 9(1): 102

Received Date: March 19, 2021 Accepted Date: June 21, 2021 Published Date: June 23, 2021

\begin{abstract}
The clinical course of our patient highlights an atypical presentation of thromboembolic disease related to pregnancy, a thrombophlebitis of the right ovarian vein with endocaval floating thrombus. The diagnosis remains difficult since it is a rare entity with an atypical presentation but with serious medical consequences.

Keywords: Thrombosis; Ovarian Vein; Postpartum; Pyrexia; Coagulation

Key Clinical Message: Thrombophlebitis of the right ovarian vein is a rare presentation of thromboembolic disease in postpartum period. Diagnostic is challenging. This case report reminds clinicians the importance of not forgetting this entity.
\end{abstract}




\section{Introduction}

Pregnancy-associated thromboembolic disease, and in particular pulmonary embolism, is a major cause of maternal morbidity and mortality in Western countries. The pathology is due in part to the natural shift of the coagulation and fibrinolysis system to a state of hypercoagulability during pregnancy, which minimizes the risk of bleeding during delivery. Other predisposing factors include maternal age, immobilization, thrombophilia, personal and family history of thrombosis, pre-eclampsia, obesity, smoking, and the mode of delivery. The immediate postpartum period is also a major risk factor. Pelvic vein thrombosis represents less than $1 \%$ of thromboses outside of pregnancy or pelvic surgery, but accounts for 10\% of thromboses during pregnancy and postpartum [1]. The diagnosis remains difficult because it is a rare entity with a mostly atypical presentation. We report the clinical management and anticoagulation therapy of a patient with a postpartum thromboembolic complication with an uncommon clinical presentation.

\section{Case presentation}

A 36-year-old woman, gravida 3, para 2, without any health problems presented to the emergency room 11 days postpartum for chills, sweating, myalgias, headache, and back pain radiating to the cervical region associated with a stiff neck. She had no associated abdominal pain but reported of strong-smelling, menstruation-type vaginal discharge. The pregnancy was unremarkable with a post-term eutocic delivery with induction of labor and birth of a healthy female child weighing 2,920 g. There was, however, a difficult epidural catheter placement. Pathological analysis of her past miscarriage did not reveal any chromosomal abnormalities in the fetus. In addition, she previously underwent a conization for grade III dysplasia and human papilloma virus infection. The patient did not have a general history of thromboembolism. However, she previously smoked one pack of 20 cigarettes a day and took contraceptive pills. The patient also had a body mass index (BMI) of 30.

On clinical examination, the patient was pyretic with a $38.6{ }^{\circ} \mathrm{C}$ temperature. Otorhinolaryngology was normal, except for pain on cervical mobilization without neck stiffness. Cardiopulmonary auscultation was normal. Abdominal examination revealed suprapubic pain and left iliac fossa pain on deep palpation. The neurological examination was unremarkable. A gynecologic ultrasound revealed only minimal fluid in the pouch of Douglas.

Blood tests revealed anemia with a hemoglobin level of $9.7 \mathrm{mg} / \mathrm{dL}$, no hyperleukocytosis, but a C-reactive protein level of 224 $\mathrm{mg} / \mathrm{L}$ and a D-Dimer level of 3,543 $\mu \mathrm{g} / \mathrm{L}$. The remaining hematology results were within normal limits. The urinalysis and blood cultures were negative. A brain scan was within normal limits. An abdominopelvic computed tomography (CT) scan with portal phase contrast revealed thrombophlebitis of the right ovarian vein over its entire length,/span> extending to the inferior vena cava in the form of a floating thrombus with a diameter of $15 \mathrm{~mm}$ (Figure 1).

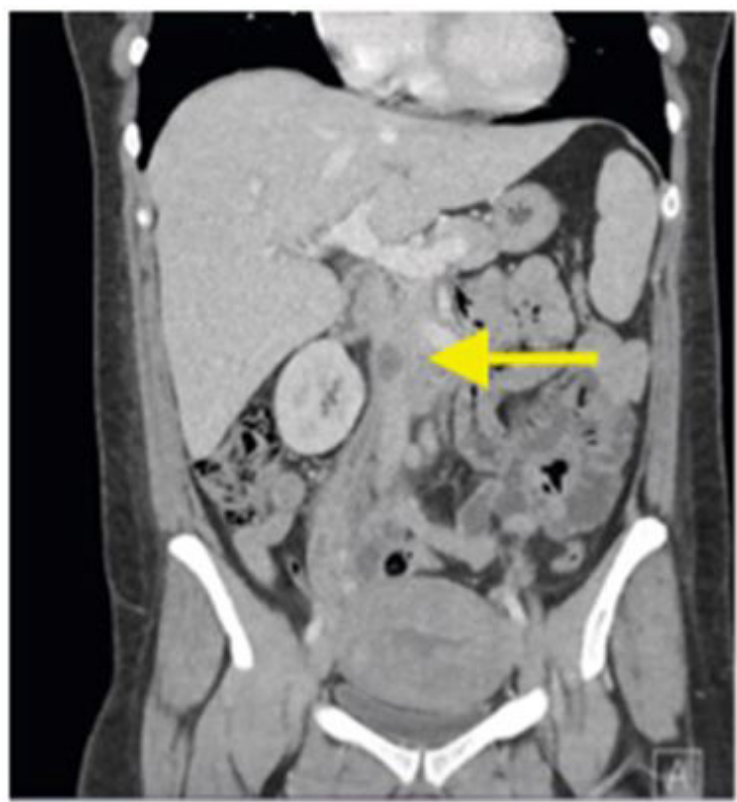

Figure 1: Computed tomography scan of the abdomen on admission shows the inferior endocaval floating thrombus Consent: I, Doctor in Medicine,hereby certify that I obtained consent from the patient for the publication of the case report 
Given the context and the patient's age, we presented our patient's case to the cardiothoracic surgeon of our hospital, who indicated an emergency vena cava filter placement via the right jugular approach under local anesthesia. The filter was deployed to the right renal vein without any incident.

Despite administration of cefazolin, the patient subsequently developed several episodes of pyrexia without an infectious focus, and several negative blood cultures. These episodes required the patient to stay in intensive care for 24 hours two days after placing the intracaval filter, and cefazolin was changed to amoxicillin-clavulanate. She then returned to the general ward and was discharged home eight days after admission. The clinical outcome was favorable. At four-month follow-up, a phleboscan and thrombophilia assessment were performed. The patient was found to be a carrier ofFactor V Leiden, and therefore prone to thromboembolic disease. The phleboscan was normal.

Subsequently, a multidisciplinary team including intensivists, surgeons, pulmonologists, and hematologists determined to treat the patient with rivaroxaban (Xarelto ${ }^{\oplus}$, Janssen Pharmaceuticals, Inc., Titusville, NJ, USA) 15 mg twice daily for 21 days followed by 20 mg once daily for six months.

\section{Discussion}

Although perinatal thromboembolic management has improved in the past 20 years, each case is critical because it concerns both, a mother and a child. This case demonstrates the difficulty of diagnosing postpartum thromboembolic diseases and the importance of considering these diagnoses given the seriousness of the complications [1]. While thromboembolic diseases are common during pregnancy and postpartum, thrombophlebitis of the ovarian vein remains a rare complication [2]. Manifestations are most often associated with pyrexia with abdominal pain. Differential diagnoses include acute appendicitis, urinary tract infection, or other pelvic conditions [1]. Furthermore, the majority of ovarian vein thromboses affect the right ovarian vein for anatomical reasons; the right ovarian vein is compressed at the level of the sacral promontory by a large dextroverted uterus with an anterograde ovarian vein flow. Finally, normal pregnancy includes changes in venous stasis, hypercoagulability, and potential venous injury, which promotes these thromboses [3], this is named Virchow's triad.

The basic treatment for thrombosis consists of antibiotic and anticoagulation therapy [4]. One of the major complications is extension to the vena cava, which was present in our patient, with the risk of pulmonary embolism. Our patient had pyrexia, but abdominal complaints were not in the foreground, which did not facilitate the diagnosis. However, the postpartum context was a reminder of this diagnostic possibility. D-dimer testing can help in the diagnosis, but the usual presence of D-dimers in the immediate postpartum can be misleading [5].

According to a well-known paper [6], in addition to the hypercoagulable state associated with pregnancy, there are various predisposing factors for postpartum thromboembolism: thrombophilia, thrombotic history in the patient and family, maternal age, pre-eclampsia, cesarean section, high BMI, (above normal for the patient), immobilization, and placental abruption. Therefore, it would be interesting to include a calculation of thromboembolic disease risk in the pre-conception gynecology or pregnancy follow-up consultations to determine which patients would benefit from preventive treatment. Unfortunately, the usual scores (Wells, Geneva) are not validated for specific populations, such as pregnancy or postpartum [7].

Considering the various reports in the literature and our own experience, we can reasonably assert that each woman presenting with fever in the postpartum period must be checked for thromboembolic disease. Unfortunately, the lack of references concerning the safety and efficacy of treatment for perinatal thromboembolic disease, and the lack of validated diagnostic scores, does not allow a comprehensive recommendation at present. Finally, all emergency professionals should keep in mind that the postpartum period may have a very high risk of thromboembolic disease, which may have an unusual presentation. This is even more critical in this particular period of COVID-19, which seems to give a state of hypercoagulability [8] whose origin is not yet known at the time of writing this paper.

\section{Conclusion}

In Western countries, thromboembolic disease is the leading cause of pregnancy-related morbidity and mortality up to six weeks postpartum

[2]. It is linked to the state of hypercoagulability associated with pregnancy as well as other predisposing factors. Thrombophlebitis of the ovarian vein is rare, and difficult to diagnose in view of its nonspecific presentation. However, it is important to highlight this possible diagnosis in view of the risks of extension to the inferior vena cava and pulmonary embolism.

\section{Author Contribution}

Main work in patient care, data collection, and manuscript writing. 


\section{References}

1. James AH(2009) Venous thromboembolism in pregnancy. Arterioscler Thromb Vasc Biol 29:326-31.

2. Tepper NK, Boulet SL, Whiteman MK, Monsour M, Marchbanks PA,et al. (2014) Postpartum venous thromboembolism: incidence and risk factors. Obstet Gynecol 123:987-96.

3. McLean K, Cushman M (2016) Venous thromboembolism and stroke in pregnancy. Hematol Am Soc Hematol Educ Program 2016: $243-50$.

4. Slim K, Chaouki M, Sana M (2011) Thrombosis of the ovarian vein in the postpartum period: an unrecognized emergency[Thrombose de la veine ovarienne dans le post-partum: une urgence méconnue]. Med Reprod 13:119-23.

5. Goodacre S, Horspool K, Nelson-Piercy C, Knight M, Shephard N, et al. (2019) The DiPEP study: an observational study of the diagnostic accuracy of clinical assessment, D-dimer and chest x-ray for suspected pulmonary embolism in pregnancy and postpartum. BJOG 126: 383-92.

6. Lindqvist PG, Torsson J, Almqvist A, Björgell O(2008) Postpartum thromboembolism: severe events might be preventable using a new risk score model.Vasc Health Risk Manag 4:1081-7.

7. Touhami O, Marzouk SB, Bennasr L, Touaibia M, Souli I, et al. (2018) Are the Wells Score and the revised Geneva Score valuable for the diagnosis of pulmonary embolism in pregnancy? Eur J Obstet Gynecol Reprod Biol 221:166-71.

8. Allegra A, Innao V, Allegra AG, Musolino C(2020) Coagulopathy and thromboembolic events in patients with SARS-CoV-2 infection: pathogenesis and management strategies. Ann Hematol 99:1953-65.

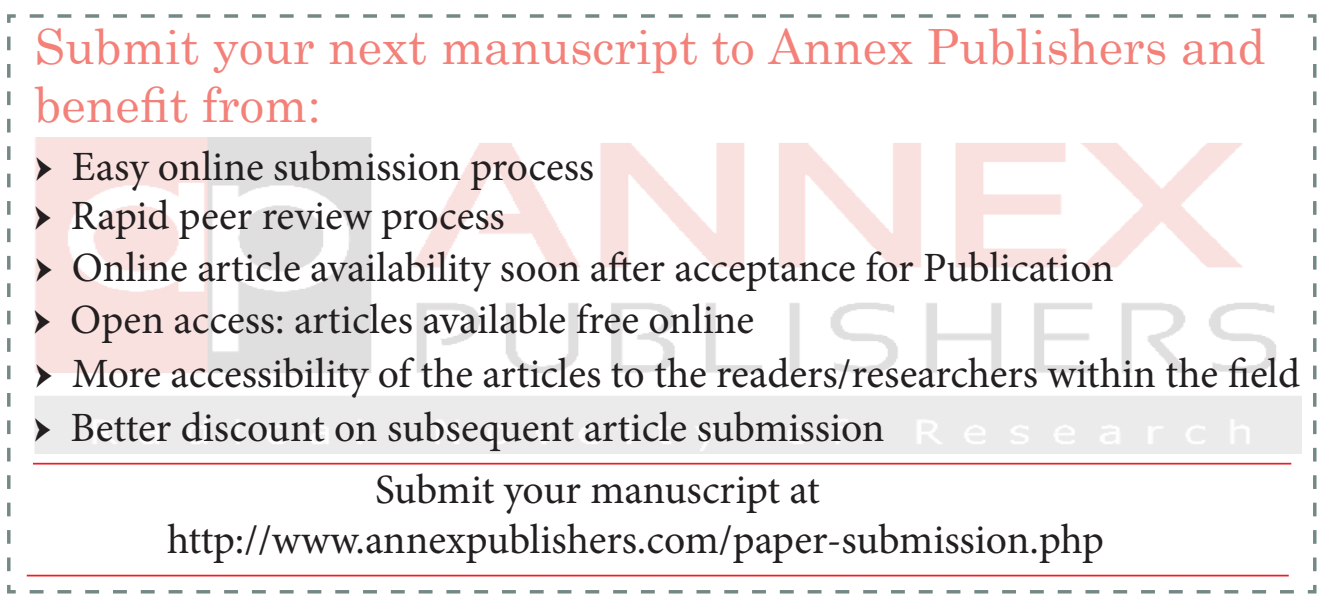

